\title{
Off-label prescribing in psychiatric practice
}

\author{
David S. Baldwin \& Nick Kosky
}

\begin{abstract}
Drug treatment is an essential part of much of psychiatric practice, in patients from a wide age range, across many diagnostic groups and in a variety of settings. Despite the availability of many classes of psychotropic drug, significant numbers of patients remain troubled by distressing and disabling symptoms even after a succession of licensed pharmacological treatments. Psychiatrists may then consider the prescription of a psychotropic outside the narrow terms of its licence, as part of an overall management plan. This article reviews the nature and extent of this aspect of prescribing, outlines when it may be appropriate and makes recommendations for a suggested procedure when prescribing medication 'off-label'.
\end{abstract}

While most authorities agree that use of drugs outside the terms of their licence is a necessary part of psychiatric practice, instances of unconventional prescribing of psychotropic drugs and worries about potentially unnecessarily restrictive policies led Professor Thomas Barnes, then Chair of the Psychopharmacology Special Interest Group (PSIG) of the Royal College of Psychiatrists, to convene a working group with the remit of examining this issue. Discussions within this group, and comments from the PSIG and wider membership of the College, led to publication of the Council Report Use of Licensed Medicines for Unlicensed Applications in Psychiatric Practice (Royal College of Psychiatrists, 2007). Our involvement in the working group led to the writing of this article.

\section{What is unlicensed prescribing?}

In the UK, 'licensed' medicines are those that have received a 'marketing authorisation' (previously called a product licence). Licensing arrangements are determined by the Medicines Act 1968 and currently implemented through the Medicines and Healthcare products Regulatory Agency (MHRA). For each medicine, the doses, indications, cautions, contraindications and side-effects given in the British National Formulary (BNF; British Medical Association
\& Royal Pharmaceutical Society of Great Britain, 2007) reflect those in the manufacturer's data sheets or summary of product characteristics (SPC), which in turn reflect the marketing authorisation. The prescription of medicines within the terms of their licence is not a guarantee of safety or efficacy. Furthermore, absence of a licence does not necessarily imply absence of evidence for an intervention. For example, low-dose propranolol has proven efficacy in treating akathisia associated with antipsychotic drugs (Adler et al, 1986) but has no licence for that condition.

Many medicines that are prescribed to patients are not licensed for the particular indication, age of the patient or dosage, and their use in these situations has been termed 'off-label' or 'unlicensed' prescribing, or the 'use of licensed drugs for an unlicensed indication'. The use of a psychotropic drug for an unlicensed indication does not necessarily imply a safety hazard, and there are many instances where use is uncontroversial and probably advantageous to the patient. The product licence for a drug does not necessarily represent the best use of that medicine (Healy \& Nutt, 1998). It may be helpful to conceptualise a spectrum of use of licensed psychotropic drugs in unlicensed applications, with some prescribing being regarded as 'near-label' (for example, use of the antidepressant fluoxetine as a maintenance treatment in a patient with recurrent depression).

\footnotetext{
David Baldwin is Reader in Psychiatry in the Clinical Neuroscience Division of Southampton University's School of Medicine (University Department of Mental Health, Royal Southants Hospital, Brintons Terrace, Southampton SO14 0YG, UK. Email: dsb1@soton.ac.uk) and Honorary Consultant Psychiatrist with the Mood Disorders Service, Hampshire Partnership Trust. He was Chair of the working group of the Royal College of Psychiatrists' Psychopharmacology Special Interest Group (PSIG) on unlicensed applications of licensed drugs in psychiatric practice. His research interests include the clinical pharmacology of anxiety and depressive disorders. He leads a tertiary referral service for patients with chronic and severe mood and anxiety disorders. Nick Kosky is Consultant Psychiatrist and Clinical Director of Dorset Primary Care Trust. He was a member of the PSIG working group. He is interested in teaching psychopharmacology to non-medical staff and delivering high-quality prescribing. He is part of a prison mental heath in-reach team.
} 


\section{What is the extent of unlicensed prescribing in psychiatry?}

Many doctors believe that off-label prescribing is often necessary when attempting to treat certain patients, for example those whose symptoms have proven resistant to a range of treatment approaches. The extent of unlicensed prescribing in psychiatric practice is not fully known, but survey findings and research studies suggest that it is common across psychiatric specialties in the UK and in many other countries. For example, in a postal questionnaire survey of 200 National Health Service (NHS) consultants, specialist registrars and staff-grade doctors in one area of the UK, almost two-thirds of doctors reported having prescribed medicines for unlicensed applications in the previous month. Most instances $(49 \%)$ involved use of a medicine outside its licensed indications, but prescriptions of drugs at doses above those described in the BNF $(19 \%)$ or outside the specified age range $(12 \%)$ were also common (LowePonsford \& Baldwin, 2000).

\section{General adult psychiatry}

Unlicensed use of licensed drugs is a common feature of prescribing in general psychiatry settings. A cross-sectional survey of prescription cards for 266 psychiatric in-patients in acute wards from 14 NHS trusts found that $7.5 \%$ of 1387 prescriptions were outside the terms of the product licence: $75 \%$ of these were for indications not covered by the licence, and $25 \%$ were at doses above the recommended maximum. In all, 81 patients were prescribed at least one medicine off-label, typically for an unlicensed indication (Douglas-Hall et al, 2001). Other investigations suggest that unlicensed prescribing may be rather more common: for example, an audit of antipsychotic drug prescribing over 5 years in a secondary care NHS trust found that about $40 \%$ of prescriptions were for off-label applications (Hodgson \& Belgamwar, 2006). Furthermore, a cross-sectional survey of prescriptions for moodstabilising drugs in 249 in-patients in a tertiary care unit found that $28.5 \%$ were receiving prescriptions for unlicensed indications (Haw \& Stubbs, 2005a).

The extent of unlicensed prescribing in UK psychiatric out-patient practice is uncertain, but research findings suggest that it is common in other European Union countries. A prospective evaluation of prescribing involving 209 out-patients in Italy found that over half of them were given off-label prescriptions of atypical antipsychotics (Barbui et al, 2002). A similar situation was found in a prescription review of 173 patients attending pharmacies in Austria, which revealed that two-thirds of them were receiving antipsychotics for unlicensed indications
(Weiss et al, 2000). Similar practice is seen in nonEuropean countries: for example, a prospective evaluation of prescribing of atypical antipsychotic drugs given to 73981 combat veterans in the USA found that $42.8 \%$ of prescriptions were for unlicensed indications (Rosenheck et al, 2001).

\section{Psychiatry of old age}

Many licensed psychotropic drugs are used for unlicensed indications when treating elderly people with mental health problems. Although at present there are no drugs specifically licensed for the treatment of psychotic and behavioural symptoms in patients with dementia, a postal questionnaire survey of 377 members of the Royal College of Psychiatrists' Faculty of Old Age Psychiatry (recently renamed the Psychiatry of Old Age) found that most doctors had used psychotropic drugs for this indication. Conventional and atypical antipsychotics were used for treating patients with delusions, hallucinations, agitation, wandering, aggression or sexual disinhibition; and antidepressants were employed in patients with anxiety and lability of mood (Scott et al, 2002). In a 1-week cross-sectional survey of 750 prescription cards for 400 elderly (aged 60-93 years) people receiving in-patient psychiatric care in 19 NHS trusts, atypical antipsychotics were prescribed to $42 \%$ of patients, of whom half had the diagnosis of a dementia (Beck et al, 2001).

Most people with dementia are unable to comprehend the reasons for using licensed drugs for unlicensed indications. Although it may be good practice to discuss a proposed unlicensed treatment with relatives or carers, it should be remembered that they do not have the right to consent to treatment on behalf of incapacitated adults. Detailed consideration of capacity is outside the scope of this article. ${ }^{\dagger}$

\section{Child and adolescent psychiatry}

Off-label prescribing to children is common in primary care settings in the UK (Ekins-Daukes et al, 2005). Psychotropic drug prescribing has become a more common aspect of practice in child and adolescent psychiatry (Bramble, 1992, 2003; McNicholas, 2001), but the full extent of unlicensed prescribing in UK child and adolescent mental health services is unknown. A questionnaire survey of community child and adolescent psychiatrists in the West Midlands found that $88 \%$ of doctors reported

\footnotetext{
${ }^{+}$Capacity to consent has been discussed previously in APT: see Bellhouse et al (2001) for consent in adults and Shaw (2001) for children and adolescents. Both of these articles can be downloaded free from http:/ / apt.rcpsych.org. Ed.
} 
issuing prescriptions for antidepressants and 63\% for antipsychotics: it can be assumed most of these prescriptions were for unlicensed indications; twothirds reported prescribing the unlicensed compound melatonin (Doerry \& Kent, 2003).

In Germany, a retrospective study of over 1.74 million prescriptions written for 400000 children by primary care physicians found that $13.2 \%$ were for unlicensed applications: substantial proportions of the prescriptions for antidepressants $(36.6 \%)$ and antipsychotics (10.2\%) were off-label (Bücheler et $a l, 2002)$. In Holland, a national survey of all child psychiatrists found that off-label prescribing was common (Hugtenburg et al, 2005). In the USA, a cross-sectional population-based study in three health maintenance programmes over 5 years found that the number of prescriptions of psychotropic drugs had increased dramatically between 1991 and 1995, many of them being for unlicensed applications (Zito et al, 2000). A study of adolescents consecutively admitted to a US private psychiatric hospital found that over half of them were prescribed antipsychotics, $73 \%$ of these prescriptions being for mood or anxiety disorders (Pogge et al, 2007). In Australia, a nationwide survey of general paediatricians and child and adolescent psychiatrists found that $40 \%$ reported off-label prescribing of psychotropics, including antidepressants, psychostimulants, antipsychotics and mood stabilisers (Efron et al, 2003).

In a consensus statement, the British Association for Psychopharmacology (1997) noted that it appears reasonable to extrapolate what is known about drug treatment responses in adults to children and adolescents in the case of schizophrenia and obsessive-compulsive disorder, but that more caution is required in the case of mood and anxiety disorders. A policy statement on the use of unlicensed medicines or licensed medicines for unlicensed applications issued by the UK Royal College of Paediatrics and Child Health (2000) makes a number of clear recommendations regarding this aspect of child healthcare.

\section{Psychiatry of intellectual disability}

Psychotropic drugs are often prescribed for unlicensed indications in people with mental health problems and behavioural challenges arising from developmental delay or arrest. Common indications include the management of sleep disturbances, increased arousal and self-injurious behaviour, and problems related to behavioural changes resulting from epilepsy syndromes and dementing disorders. A cross-sectional survey of psychotropic drug prescribing in in-patients with intellectual disability found that $46.4 \%$ were receiving at least one psychotropic for an unlicensed indication, most typically in an attempt to manage behavioural problems or to stabilise mood (Haw \& Stubbs, 2005b).

The dearth of randomised controlled trials in this patient population means that most prescriptions are outside of product licences, although lithium is licensed for the management of aggressive or self-mutilating behaviour. Most of the common interventions are supported by retrospective case series analysis only. A review of the use of atypical antipsychotics in people with autistic-spectrum disorders reflects current thinking in the wider field of developmental neuropsychiatry and intellectual disability (Barnard et al, 2002). Uncertainties regarding diagnosis and capacity to consent, and associated physical health problems, must all be considered when formulating potential treatment approaches (British Association for Psychopharmacology, 1997) and psychotropic drug prescription is usually only one component of a multifaceted management approach.

\section{Forensic psychiatry}

The unlicensed use of psychotropic drugs in forensic psychiatry settings can be especially contentious, especially as randomised controlled trials provide minimal evidence for greater efficacy of 'mega-doses' over standard doses in antipsychotic drug treatment. A report on the use of high-dose antipsychotic medication (Royal College of Psychiatrists, 2006) provides guidance on when doses higher than those described within the BNF may be indicated. The practice of issuing 'as required' prescriptions on top of regular administration may result in overall daily dosages in excess of current recommendations (Milton et al, 1998). Similar concerns apply to concurrent prescription of oral and depot antipsychotic drugs, or concomitant prescription of conventional (typical) antipsychotics with atypical antipsychotics. Treatment considerations should be guided not only by the Mental Health Act 1983 and its supporting Code of Practice, but also by specific case law.

\section{Perinatal psychiatry}

No psychotropic medication is licensed for use in pregnancy or in breastfeeding mothers, and the $B N F$ and product information sheets advise at least caution (and in most cases contraindication) in their prescription. However, prescribing psychotropic medication is commonplace in women of reproductive age and up to $27 \%$ of women with a psychiatric disorder are receiving psychotropic medication when their pregnancies are first detected (Rubin et al, 1986; Williams et al, 1998). Although the onset of schizophrenia or bipolar disorder is less common during pregnancy than at other 
times, significant numbers of women with preexisting serious mental illness will require treatment during pregnancy. Withdrawal of medication when pregnancy is detected is associated with the usual rates of relapse or recurrence of illness, particularly in bipolar affective disorder. Therefore, psychiatrists will sometimes find it necessary to prescribe psychotropic medication for new episodes of illness as well as continue to manage women with preexisting disorders during their pregnancies.

These problems are complicated by the variety of sources of information on risks associated with medication. These include data on request from the manufacturers, prescribing information leaflets, the $B N F$, advice from professional bodies and the National Teratology Information Service, review articles and individual publications of case series. Many thousands of exposed pregnancies will need to be studied over time before any significantly associated risk can be demonstrated compared with the unexposed control population, and underpowered studies may either give false reassurance or be unduly alarmist. Results expressed in terms of relative risk with a given drug need to be viewed in context of the baseline risks in an unexposed group of pregnant women.

Studies of the effects of drugs on the rates of major congenital malformations identified soon after birth are more numerous than those describing minor malformations or neurodevelopmental problems that may be delayed in their manifestation. The evidence base changes and may take some time to be disseminated into psychiatric journals. For example, accumulating evidence of the additional hazards (over other anticonvulsant mood stabilisers) of valproate has been published largely in paediatric and neurology journals for 20 years, but this has only recently entered psychiatrists' awareness. A further problem is that evidence on the potential hazards of medication, particularly from conference proceedings, may be publicised in the media before publication in scientific journals. As the nature of the evidence continues to change, it is not wise to make categorical recommendations for one drug as opposed to another or to talk about psychotropic medications in terms of overall statements of safety or risk. The general principles of prescribing during pregnancy are described in Box $1 .^{\ddagger}$

\section{What are the main examples of unlicensed prescribing?}

There are four main types of unlicensed prescribing, relating to the medical disorder and demographics of the patient, and the dosage and duration of treatment (the 'four Ds').

\section{The disorder}

The first type is perhaps the best known and involves the prescription of a medication for an indication that is not covered within the terms of the marketing authorisation. However, new indications for existing treatments appear regularly, as shown by the expansion of indications for some of the selective serotonin reuptake inhibitors (SSRIs), so what is unlicensed prescribing one month may come within the terms of the marketing authorisation the next. Conversely, drugs may 'lose' an indication as new clinical data emerge and become available to regulatory bodies; an example of this is changes to the licensing of SSRIs (other than fluoxetine) for the treatment of depression or obsessive-compulsive disorder in children and adolescents. A different example is the removal in the UK of premenstrual dysphoric disorder from the licensed indications for fluoxetine, as a result of the harmonisation of the summary of product characteristics for fluoxetine with Europe (the indication persists in the USA).

\section{The demographics}

The second type of unlicensed prescribing involves a drug being given to a patient who is outside the age range specified within the summary of product characteristics. The March 2007 BNF states that prescribing the noradrenaline reuptake inhibitor reboxetine to children and elderly patients is 'not recommended' (British Medical Association \& Royal Pharmaceutical Society of Great Britain, 2007) (even though a randomised controlled trial has documented its efficacy in the treatment of depression in elderly patients). For instance, prescription of reboxetine to a 65-year-old patient is licensed, but it becomes unlicensed when they turn 66 , even though it is unlikely that they will differ much in their metabolism and response to antidepressant treatment when they cross the threshold of their 66th birthday. When selecting patients for inclusion in a drug trial it is common practice to set an arbitrary upper age limit (usually around 70 years). Exclusion of adolescents or children is also routine, although in this case considerations of capacity to give consent, as well as differences in pharmacokinetics, make this more defendable. All of the available SSRIs have proven efficacy in the treatment of obsessive-compulsive disorder, and some have efficacy in randomised controlled trials conducted in children and adolescents. The March 2007 BNF notes that two SSRIs (fluvoxamine, aged 8 years and over; sertraline,

$\ddagger$ Psychotropic medication in pregnancy has been discussed in more detail in APT by Kohen (2004). Ed. 


\section{Box 1 General principles for prescribing of psychotropic drugs during pregnancy}

- Wherever possible, women receiving psychotropic medication should carefully plan their pregnancies and discuss with their general practitioner and psychiatrist prior to conception whether to continue, change or stop their medication. This is particularly important for women receiving treatment for serious mental illness and where there may be a significant risk of relapse following cessation of medication.

- Unless there are positive reasons not to do so (for example evidence of previous relapse when changing medication), medication should be changed prior to conception to that which has the best safety profile in pregnancy.

- In general, because they have been in use for many years, there is more information available about the effects of older drugs compared with newer ones.

- Wherever possible, medication should be avoided in the first trimester. However, if women conceive while taking psychotropic medication it should not be abruptly withdrawn. A relapse of the maternal condition may involve increased risks through treating the relapse.

- Mild non-psychotic conditions are common in pregnancy and wherever possible psychological treatments are preferable to psychotropic medication. The threshold for prescribing psychotropic medication in pregnancy should be high and based on clear indications.

- While the incidence of serious mental illness in pregnancy is lower than at other times, when these illnesses occur they should be treated energetically. The risk to both mother and the unborn child of not treating the illness may be higher than the potential risks to the unborn child of the medication.

- Pregnancy is not protective against a relapse of serious mental illness, particularly if medication has been stopped. Continuing medication is therefore in the best interests of both the mother and the infant. However particular attention needs to be paid to the choice of individual antipsychotic and mood stabilising agents.

- Psychiatrists, obstetricians and neonatal paediatricians should be aware of the potential of withdrawal effects in neonates if the mother has been taking antidepressants or antipsychotic medication prior to delivery (Sanz et al, 2005).

- The lowest dose possible should be used in divided dosage and polypharmacy avoided.

- Doctors prescribing psychotropic medication to pregnant and breastfeeding women should ensure that they have the most up to date information possible. A good source of balanced and regularly updated information can be obtained from The National Teratology Information Service (see their website at http:/ / www.nyrdtc.nhs.uk).

- Doctors should always work in partnership with the women and their partners and be able to discuss the risks and benefits of their medication in a way that is easily understood.

- When prescribing medication in pregnancy or lactating women, the psychiatrist should clearly document the reasons why the medication is being prescribed, an indication that the risks and benefits have been addressed, that the woman has been involved in the decision and a note made of any advice or information received that has influenced the decision and choice of medication.

(Royal College of Psychiatrists, 2007: pp. 15-16)

6 years and over) can be used in the treatment of children with the disorder (British Medical Association \& Royal Pharmaceutical Society of Great Britain, 2007).

\section{The dose}

Another type of unlicensed prescribing is the use of a medicine outside the dose range recommended in the summary of product characteristics and reflected in the BNF. High-dose antidepressants (e.g. venlafaxine at more than $375 \mathrm{mg}$ a day) are sometimes recommended by tertiary services in the management of treatment-resistant depression; similarly, supra$B N F$ dosing with antipsychotics is not uncommon in forensic practice, especially in the management of treatment-resistant psychosis. However, the effectiveness of these strategies is uncertain.

\section{The duration}

There is another type of unlicensed prescribing, representing the use of a licensed medication for longer periods than those specified within the 
marketing authorisation. For example, most antidepressants are licensed for treating 'depressive illness'. Continuation and maintenance treatment with antidepressants of people with recurrent depressive disorder when they are asymptomatic and in remission might technically therefore represent unlicensed use, but also clearly represents an aspect of good clinical practice. The Committee on Safety of Medicines currently advises that prescription of benzodiazepines should be limited to 4 weeks only (reflected in Section 4.1 of the BNF; British Medical Association \& Royal Pharmaceutical Society of Great Britain, 2007), but many people with chronic and disabling anxiety disorders who have not responded to other treatments may benefit from longer courses of treatment (Haw \& Stubbs, 2006).

\section{What is the legal position regarding unlicensed prescribing?}

In Britain there is no statutory requirement to disclose to the patient when a drug prescription is unlicensed, but prescribing of licensed medicines outside the recommendations of the marketing authorisation alters and probably increases professional liability (Anonymous, 1992). When prescribing outside these recommendations the doctor must be able to justify this action in accordance with a respectable, responsible body of professional opinion. Increasing emphasis on risk management and evidence-based practice might encourage some trusts to introduce systems and protocols for monitoring and possibly directing the use of psychotropic drugs, and some trusts have suggested that doctors should not use licensed medicines for unlicensed applications. However, in 1993 the Department of Health stated that it would not expect that a health authority would seek to fetter a clinician's freedom to prescribe by expressly directing its medical staff against unlicensed prescribing (Anonymous, 1992). In the same article it is noted that the Department of Health's lawyers also stated that, should a health authority so direct its medical staff, a Court would be reluctant to support the authority in those circumstances.

In general, the Courts would not hold unlicensed prescribing to be a breach of the duty of care, provided that treatment was supported by a respected body of medical opinion, as the Bolam test in medical negligence claims asks for proof that a body of doctors would act similarly to the doctor in question (Bolam v. Friern Hospital Management Committee, 1957). In addition, the more recent Bolitho case (Bolitho v. City and Hackney Health Authority, 1997) states that medical opinion should also be capable of withstanding logical analysis, which in this instance would imply that doctors should consider the risks and benefits of various treatment options, with regard to the evidence that is available and the nature of the clinical case.

More information on the legislation for licensing of drugs can be found on the websites of the European Agency for the Evaluation of Medicinal Products (http://www.emea.europa.eu) and the International Conference on Harmonisation of Technical Requirements for Registration of Pharmaceuticals for Human Use (http://www.ich.org). A thoughtful consideration of current drug regulatory policies is available elsewhere (Garattini \& Bertele, 2005). In the UK, the Medicines Act 1968 and the Medicines Act 1968 (Amendment) Regulations 1992 are no longer the source of the control in dealing with medicinal products, these matters being addressed by Council Regulation (EEC) no. 2309/93 (laying down procedures for the authorisation and supervision of medicinal products).

\section{What procedures should be adopted when prescribing for unlicensed applications?}

A number of groups have sought to develop mechanisms for evaluating and ensuring the appropriateness of off-label prescribing of medicines (Ansani et al, 2006; Gazarian et al, 2006). The Royal College of Psychiatrists' recommendations are shown in Box 2. Clearly, a balance has to be struck between undue therapeutic conservatism that limits patient choice and reduces the chance of optimal clinical outcomes, and overenthusiastic or idiosyncratic approaches that deny patients access to the best evidence-based treatments. Where a proposed application could be considered hazardous, it would be advisable to adhere closely to the suggestions listed in Box 2; however, elaborate measures are less important where the proposed treatment is 'nearlabel' and not controversial or particularly novel.

As in all aspects of evidence-enhanced healthcare, treatment decisions should not be based solely on evidence from randomised controlled trials: other influences, such as the patient's preferences, clinical judgement and local service availability, are also important. Many accepted medical interventions are supported not by evidence of efficacy from randomised placebo-controlled trials but by the evidence of clinical effectiveness over many years. The restriction of prescribing to licensed indications or to clinical situations with evidence from randomised controlled trials may unwittingly inhibit the use of effective drugs from an earlier era of drug registration.

Whenever possible, potential approaches to treatment should be considered with due regard to the 


\section{Box 2 Suggested procedure when prescribing medication 'off-label'}

1 Check that medicines with a product licence have either had a proper therapeutic trial or been considered, but excluded on clinical grounds (such as contraindications and risk of interactions).

2 Familiarise yourself with the evidence about the proposed drug, including any possible drug interactions and potential adverse effects.

3 If the medicine to be used does not have a substantial evidence base supporting its use for the proposed indication, or if you are not sufficiently expert in this field, or have particular concerns, obtain the advice of another doctor or specialist pharmacist.

4 Consider the risks and benefits of the proposed treatment. Particular consideration is needed with children, older patients, and in those with impaired insight and judgement. Document this.

5 Give the patient (or his/her relative, when relevant) a full explanation, including the information that the drug will be used outside its product licence. Document this explanation.

6 If agreement from the patient (or his/her relative, when needed) is obtained, document this approval. If a patient is unable to consent to a necessary treatment, note that it has not been possible to obtain consent.

7 Begin a cautious trial of treatment with the medicine. In out-patients, consider sending the patient a copy of any letter sent to his/her general practitioner, summarising why this approach has been adopted.

8 Monitor the patient closely. Continue with full documentation of its effectiveness and tolerance.

9 If the treatment proves unsuccessful, withdraw it, gradually if needed. Document the reason for the withdrawal of treatment, then consider alternatives, using the same process.

10 Consider writing up the case, to add to knowledge about the drug and its use.

(Royal College of Psychiatrists, 2007: pp. 6-7)

anticipated benefits and risks, and discussed fully with the patient and, if appropriate, with family members. Treatment decisions do not usually need to be rushed, and the reasons for a change in approach can be adequately documented in the medical notes. In especially urgent clinical situations (for example, the need for rapid tranquillisation of someone with a behavioural disorder experiencing an acute psychotic episode), some steps may need to be taken quickly, but even here other treatment approaches involving the licensed use of medicines can often be instituted while the possible unlicensed use of medicines is considered.

In situations where most prescribing is for unlicensed indications (for example, in many aspects of practice in old age psychiatry) it is simply not possible to fully document the reasons for every prescribing decision in every patient. The absence or non-availability of surviving relatives can make it impossible to discuss particular aspects of prescribing practice with a carer and in this situation doctors might wish to document when and why it has not been possible to consult relatives.

General practitioners are involved in the continuing care of psychiatric out-patients and often have input to the management of their patients undergoing in-patient psychiatric care. It is probably best for psychiatrists to liaise with their primary care colleagues at the time of instituting a new unlicensed treatment for an out-patient and before discharge from hospital of an in-patient. Most general practitioners would be prepared to implement a treatment recommendation from a colleague, provided the rationale and practical arrangements have been clarified. A similar situation applies when doctors working in tertiary referral specialist centres make treatment recommendations to colleagues working in more standard settings.

\section{Are there wider implications?}

Pharmaceutical companies typically evaluate potential new medicines in patients aged between 18 and 65 years, and there is no statutory requirement that drugs are tested and licensed specifically in other populations, such as children and elderly people. Consequently, the data about a drug when it first becomes available are usually insufficient to inform its potential use in patients at the extremes of the age distribution. Doctors working with patients from these age groups are therefore placed in a difficult position, and might benefit from discussing these matters within peer groups or across specialties, to clarify their position on unlicensed prescribing. 
The process of obtaining modifications to a product licence is lengthy and costly, and pharmaceutical companies may be deterred from pursuing potential new indications for an already available drug, particularly when its patent is soon to expire. By contrast, doctors become prepared to use tried and tested treatments in potential new clinical applications, similar to those for which the product already has a licence. In these situations, there is much scope for data collection and local pharmacovigilance, but this requires the support of medicines management committees and the provision of other trust resources. It is important that these novel uses in case series of patients are submitted for scientific publication, as the resulting generation of an evidence base allows doctors to cite 'custom and practice' on the effectiveness and acceptability of proposed treatments when there is no evidence of efficacy from randomised controlled trials.

\section{Acknowledgement}

We wish to acknowledge the support and encouragement of the late Professor Robert Kerwin.

\section{Declaration of interest}

D. S. B. has acted as a consultant to a number of companies with an interest in anxiety and depressive disorders (Asahi, Bristol-Myers Squibb, Cephalon, Eli Lilly, GSK, Lundbeck, Organon, Pfizer, Pharmacia, Pierre Fabre, Roche, Servier, Sumitomo, Wyeth) and holds or has held research grants (on behalf of his employer) from Bristol-Myers Squibb, Cephalon, Eli Lilly, GSK, Lundbeck, Organon, Pfizer, Pharmacia, Roche and Wyeth. N. K. has acted as consultant and trainer to companies marketing antidepressants and antipsychotics (Bristol-Myers Squibb, Eli Lilly, Janssen-Cilag, Lundbeck, Wyeth) and has conducted sponsored research or held research grants (on behalf of his employer) from Bristol-Myers Squibb, GSK and Janssen-Cilag.

\section{References and further reading}

Adler, L., Angrist, B., Peselow, E., et al (1986) A controlled assessment of propranolol in the treatment of neurolepticinduced akathisia. British Journal of Psychiatry, 149, 42-45.

Anonymous (1992) Prescribing unlicensed drugs or using drugs for unlicensed indications. Drug and Therapeutics Bulletin, 30, 97-99.

Ansani, N., Sirio, C., Smitherman, T., et al (2006) Designing a strategy to promote safe, innovative off-label use of medications. American Journal of Medical Quality, 21, 255-261.

Barbui, C., Danese, A., Guaiana, G., et al (2002) Prescribing second-generation antipsychotics and the evolving standard of care in Italy. Pharmacopsychiatry, 35, 239-243.
Barnard, L., Young, A. H., Pearson, J., et al (2002) Systematic review of the use of atypical antipsychotics in autism. Journal of Psychopharmacology, 16, 93-101.

Beck, S., Paton, C., Euba, R., et al (2001) Atypical antipsychotics in the elderly. International Journal of Psychiatry in Clinical Practice, 5, 257-261.

Bellhouse, J., Holland, A., Clare, I., et al (2001) Decision-making capacity in adults: its assessment in clinical practice. Advances in Psychiatric Treatment, 7, 294-301.

Bradley, E. \& Lofchy, J. (2005) Learning disability in the accident and emergency department. Advances in Psychiatric Treatment, 11, 45-57.

Bramble, D. (1992) The use of antidepressants by British child psychiatrists. Psychiatric Bulletin, 16, 396-398.

Bramble, D. (2003) The use of psychotropic medications in children: a British view. Journal of Child Psychology and Psychiatry, 44, 169-179.

British Association for Psychopharmacology (1997) BAP consensus statement. Child and learning disability psychopharmacology. Journal of Psychopharmacology, 11, 291-294.

British Medical Association \& Royal Pharmaceutical Society of Great Britain (2007) British National Formulary (March issue). BMJ Publishing Group \& RPS Publishing.

Bücheler, R., Schwab, M., Mörike, K., et al (2002) Off label prescribing to children in primary care in Germany: retrospective cohort study. BMJ, 324, 1311-1312.

Doerry, U. A. \& Kent, L. (2003) Prescribing practices of community child and adolescent psychiatrists. Psychiatric Bulletin, 27, 407-410.

Douglas-Hall, P., Fuller, A. \& Gill-Banham, S. (2001) An analysis of off-licence prescribing in psychiatric medicine. Pharmaceutical Journal, 267, 890-891.

Efron, D., Hiscock, H., Sewell, J. R., et al (2003) Prescribing of psychotropic medications for children by Australian paediatricians and child psychiatrists. Pediatrics, 111, 372-375.

Ekins-Daukes, S., Helms, P. J., Taylor, M. W., et al (2005) Offlabel prescribing to children: attitudes and experience of general practitioners. British Journal of Clinical Pharmacology, 60, 145-149.

Garattini, S. \& Bertele, V. (2005) The impact of European regulatory policies on psychotropic drug prescribing patterns. International Review of Psychiatry, 17, 199-204.

Gazarian, M., Kelly, M., McPhee, J. R., et al (2006) Off-label use of medicines: consensus recommendations for evaluating appropriateness. Medical Journal of Australia, 185, 544-548.

Haw, C. \& Stubbs, J. (2005a) A survey of the off-label use of mood stabilizers in a large psychiatric hospital. Journal of Psychopharmacology, 19, 402-407.

Haw, C. \& Stubbs, J. (2005b) A survey of off-label prescribing for inpatients with mild intellectual disability and mental illness. Journal of Intellectual Disability Research, 49, 858-864.

Haw, C. \& Stubbs, J. (2006) Benzodiazepines - a necessary evil? A survey of prescribing at a specialist UK psychiatric hospital. Journal of Psychopharmacology, published online. doi:10.1177/ 0269881106072386.

*Healy, D. \& Nutt, D. (1998) Prescriptions, licence and evidence. Psychiatric Bulletin, 22, 680-684.

Hodgson, R. \& Belgamwar, R. (2006) Off-label prescribing by psychiatrists. Psychiatric Bulletin, 30, 55-57.

Hugtenburg, J. G., Heerdink, E. R. \& Tso, Y. H. (2005) Psychoactive drug prescribing by Dutch child and adolescent psychiatrists. Acta Paediatrica, 94, 1484-1487.

Kohen, D. (2004) Psychotropic medication in pregnancy. Advances in Psychiatric Treatment, 10, 59-66.

Lowe-Ponsford, F. \& Baldwin, D. S. (2000) Off-label prescribing by psychiatrists. Psychiatric Bulletin, 24, 415-417.

McNicholas, F. (2001) Prescribing practices of child psychiatrists in the UK. Child Psychology and Psychiatry Review, 1, 94-97.

Milton, J., Lawton, J., Smith, M., et al (1998) Hidden high-dose antipsychotic prescribing: effects of p.r.n. doses. Psychiatric Bulletin, 22, 675-677.

Pogge, D. L., Young, K., Insalaco, B., et al (2007) Use of atypical antipsychotic medications in adolescent psychiatric inpatients: a comparison with inpatients who did not receive antipsychotic medications during their stay. International Journal of Clinical Practice, 61, 896-902. 
Rosenheck, R., Leslie, D. \& Sernyak, M. (2001) From clinical trials to real-world practice: use of atypical antipsychotic medication nationally in the Department of Veterans Affairs. Medical Care, 39, 302-308.

Royal College of Paediatrics and Child Health (2000) The Use of Unlicensed Medicines or Licensed Medicines for Unlicensed Applications in Paediatric Practice. Policy Statement Produced by the Joint RCPCH/NPPG Standing Committee on Medicines. Royal College of Paediatrics and Child Health.

* Royal College of Psychiatrists (2006) Consensus Statement on High-Dose Antipsychotic Medication (Council Report CR138). Royal College of Psychiatrists.

* Royal College of Psychiatrists (2007) Use of Licensed Medicines for Unlicensed Applications in Psychiatric Practice (College Report CR142). Royal College of Psychiatrists.

Rubin, P. C., Craig, G. F., Gavin, K., et al (1986) Prospective survey of use of therapeutic drugs, alcohol and cigarettes during pregnancy. $B M J, 292,81-83$.

Sanz, E. J, De-Las-Cuevas, C., Kuru, A., et al (2005) Selective serotonin reuptake inhibitors in pregnant women and neonatal withdrawal syndrome: a database analysis. Lancet, 365, 482-487.

Scott, K., Lawrence, R. M., Duggal, A., et al (2002) Prescribing patterns for psychotic and behavioural symptoms in dementia: a national survey. Psychiatric Bulletin, 26, 288-290.

Shaw, M. (2001) Competence and consent to treatment in children and adolescents. Advances in Psychiatric Treatment, 7, 150-159.

Weiss, E., Hummer, M., Koller, D., et al (2000) Off-label use of antipsychotic drugs. Journal of Clinical Psychopharmacology, 20, 695-698.

Williams, L. V., Pearce, G. L., Martin, R. M., et al (1998) The outcomes of pregnancy in women exposed to newly marketed drugs in general practice in England. British Journal of Obstetrics and Gynaecology, 105, 882-889.

Zito, J. M., Safer, D. J., dosReis, S., et al (2000) Trends in the prescribing of psychotropic medications to preschoolers. JAMA, 283, 1025-1030.

Bolam v. Friern Hospital Management Committee [1957] WLR, 582.

Bolitho v. City and Hackney Health Authority [1997] 3WLR, 1151.

\section{MCQs}

1 Off-label prescribing of psychotropic drugs:

a is uncommon in psychiatric practice

b can routinely be done without seeking express consent from the patient

c can result in a better outcome for the patient

d most of the time has little evidence to support it

e is not necessary outside specialist centres.

\section{Licensing a medicine:}

a involves recognition of all of its therapeutic applications

$\mathrm{b}$ is implemented by the Committee on Safety of Medicines

c limits the claims a manufacturer can make for it

d means that the best use of it has been recognised

e only happens when it first comes onto the market.
3 Prescribing psychotropic drugs in pregnancy:

a should usually involve newer drugs as they are safer than older ones

b should rarely result in a change of drug or dosing regimen

c is straightforward and needs no special consideration

d involves considerations of consent that are no different from those for a non-pregnant patient

e needs a careful consideration of possible risks and benefits to mother and baby.

4 Prescribing off-label drugs to older patients with dementia and depression:

a does not require any special precautions

b can be done without the need to consider consent

c is supported by a substantial evidence base

$\mathrm{d}$ is common

e commonly involves different medications to those used in the working-age population.

5 When prescribing off-label:

a there is no greater need to explore the evidence base than when prescribing a licensed indication

$\mathrm{b}$ there is no need to monitor the patient more closely

c there are times when written consent from the patient should be sought

$\mathrm{d}$ it is reasonable to expect primary care prescribers to continue the prescription without any particular communication about rationale

e no particular attention needs to be paid to the documentation of effectiveness and tolerability.

\section{MCQ answers}

$\begin{array}{llllllll}1 & & 2 & & 3 & & & 5 \\ \text { a F } & \text { a F } & \text { a F } & \text { a F } & \text { a F } \\ \text { b F } & \text { b F } & \text { b F } & \text { b F } & \text { b F } \\ \text { c T } & \text { c } \text { T } & \text { c F } & \text { c F } & \text { c T } \\ \text { d F } & \text { d F } & \text { d F } & \text { d T } & \text { d F } \\ \text { e F } & \text { e F } & \text { e T } & \text { e F } & \text { e F }\end{array}$

